\title{
End State Copying by Humans (Homo sapiens): Implications for a Comparative Perspective on Cumulative Culture
}

\author{
Christine A Caldwell ${ }^{1}$ \\ Kerstin Schillinger ${ }^{1}$ \\ Cara L. Evans ${ }^{1}$ \\ Lydia M. Hopper ${ }^{2}$ \\ ${ }^{1}$ Psychology, School of Natural Sciences, University of Stirling
${ }^{2}$ Language Research Center and Department of Psychology, Georgia State University.
}

It has been proposed that the uniqueness of human cumulative culture may be attributable to humans' greater orientation towards copying the process of behavior (imitation), compared with the products (emulation), resulting in particularly high fidelity transmission. Following on from previous work indicating that adult human participants can exhibit cumulative learning on the basis of product copying alone, we now investigate whether such learning involves high fidelity transmission. Eighty adult human participants were presented with a task previously shown to elicit cumulative learning under experimental conditions, which involved building a tower from spaghetti and modeling clay. Each participant was shown two completed towers, ostensibly built by previous participants, but actually built to pre-specified designs by the experimenter. This end state information was provided either in the form of photographs, or the presence of actual towers. High fidelity matching to these end states was apparent in both demonstration conditions, even for a design that was demonstrably suboptimal with regard to the goal of the task (maximizing tower height). We conclude that, although high fidelity transmission is likely to be implicated in cumulative culture, action copying is not always necessary for this to occur. Furthermore, since chimpanzees apparently copy behavioral processes and well as products, and also transmit behavior with high fidelity, the stark absence of unequivocal examples of cumulative culture in nonhumans may be attributable to factors other than imitative ability.

\section{Introduction}

Many nonhuman species have been proposed to exhibit culture, in the sense of behavioral variation that is acquired and maintained by social learning (Laland \& Galef, 2009). However, most researchers are agreed that there are striking differences between the relatively simple, and somewhat rare, cultural behaviors observed in nonhuman animals, compared with the complexity and pervasiveness of human culture (e.g. Hill, Barton \& Hurtado, 2009; Whiten, 2011). In this article we focus in particular on the phenomenon of cumulative culture, a process by which cultural change accumulates over many generations (Boyd \& Richerson, 1996), resulting in what Tomasello (1999) has described as a 'ratchetlike' effect, whereby favorable variants are typically preserved in the population until further enhancements are developed.

Even amongst our closest evolutionary relatives, chimpanzees and other apes, there is little evidence of cumulative culture. Tennie, Call and Tomasello (2009) have contended that the cultural behaviors observed in chimpanzees are, "all things that individual chimpanzees could invent on their own fairly readily if all of the external and internal conditions were 
right." (p2407), thereby discounting the possibility of cumulative change to behavior as a consequence of additive social learning. Although others have argued that there is some evidence of cumulative culture in chimpanzees (e.g. see Sanz, Call \& Morgan, 2009, for compelling examples of complex, multi-stage tool-set use by wild chimpanzees), it is nonetheless accepted that such examples are rare, and that they still fall far short of the elaborateness of human culture (Whiten, 2011).

One potential explanation for the apparent discontinuity between socially learned behaviors in humans and those of other species concerns the underlying learning mechanisms involved. A recent and detailed account of this view has been provided by Tennie et al. (2009). Tennie et al. (2009) proposed that human copying is primarily focused on bodily actions (processes), whereas the copying of other great apes is focused on physical effects or outcomes (products). The human focus on actions, they have argued, allows for faithful behavior transmission, and this high fidelity copying is suggested to be essential for cumulative culture to occur (see also Tomasello, 1999). We consider this to be a valuable proposal, but we believe that it may not be sufficient to explain the stark differences between human cumulative culture and nonhuman primate culture. In the current article we consider the various inferences on which this proposal rests. We begin by discussing the evidence for chimpanzees as process-oriented when it comes to copying, and also analyze the support for high fidelity behavior diffusion in chimpanzees. We then consider the evidence for nonimitative (i.e. product-oriented) learning supporting high fidelity transmission, and discuss the support for non-imitative learning supporting cumulative culture. In addition, we present a new study, in which we investigate whether non-imitative cumulative culture in humans involves high fidelity transmission.

\section{Imitation and Emulation as Copying of Processes and Products}

First we begin with some definitions of the main learning mechanisms that we discuss in the current article. Imitation, as most have defined it, represents copying of actions, that is, process-oriented copying. Whiten (Whiten \& Ham, 1992; Whiten, 2011), for example, has defined imitation as, "copying the form of an action". This contrasts with product-oriented copying, generally referred to as emulation (Wood, 1989). In learning a new skill through emulative learning, there are a number of possible sources of information (Byrne, 1998), and correspondingly there a number of subcategories of emulation. Whiten (2011) recently distinguished three distinct types of emulation: object movement re-enactment (recreation of the movement of objects manipulated by another individual, as opposed to the actions that brought those about); end state emulation (recreation of the end state of another's actions by any behavioral means); and affordance learning (learning about the physical properties of the objects through observation of another individual's interaction with them, facilitating the achievement of a similar goal). In the current article we subscribe to these same definitions of imitation (process-oriented copying) and emulation (product-oriented copying).

Clearly defining imitation and emulation is particularly important when considering the arguments that have been put forward to explain the rarity of cumulative culture in species other than humans. Experimental comparisons have been drawn between chimpanzees and humans (typically young children), some of which have suggested that humans predominantly copy actions, whereas chimpanzees tend to reproduce only the end states of demonstrations (e.g. Call, Carpenter \& Tomasello, 2005; Tennie, Greve, Gretscher \& Call, 2010). Such differences have led some researchers to attribute the distinctiveness of human cumulative culture to such differences in imitative proclivity. Indeed, Tomasello (1999) has gone so far as to state that, "cumulative cultural evolution depends on imitative learning, and perhaps active instruction on the part of adults, and cannot be brought about by means of "weaker" forms of social learning such as local enhancement, emulation learning, 
ontogenetic ritualization, or any form of individual learning" (Tomasello, 1999, p39; see also Tomasello, Kruger \& Ratner, 1993).

A variety of experimental methods have been used to distinguish relative reliance on imitation and emulation in social learning tasks. Two of the most prominent involve specially designed controls, referred to as "end state" and "ghost display" conditions (Hopper, 2010). In an end state control condition (e.g. Meltzoff, 1985; Call et al., 2005; Zohar \& Terkel, 1991), naïve observers are shown the completed form of a task, and sometimes the initial state too, but no information is provided about the methods used to reach that end state. Such a condition therefore provides a control for end state emulation, and also limited affordance learning. Tomasello (1998) illustrated emulation with the following example: "if a mother [chimpanzee] rolls a log and eats the insects underneath, her child will very likely follow suit ... the youngster would have learned the same thing if the wind, rather than the mother, had caused the log to roll over and expose the ants." (p. 29). The aim of ghost control experiments is to mimic just such a scenario and, accordingly, in a ghost control condition the objects move as if manipulated by a demonstrator, but crucially, this occurs without another individual actually performing those actions. This condition therefore provides a control for all three of the subcategories of emulation detailed above: end state emulation, affordance learning, and object movement re-enactment.

\section{Experimental Studies Distinguishing Imitation and Emulation in Chimpanzees}

Although some experiments using these methods have suggested that human children may copy actions better than chimpanzees (e.g. Call et al., 2005, mentioned above), we do not agree with the confident characterization of chimpanzees as product-focused, compared with process-focused humans. Our own research has shown that human children and chimpanzees will learn a challenging tool-use task much more effectively from live models than from a ghost display (Hopper, Spiteri, Lambeth, Schapiro, Horner \& Whiten, 2007; Hopper, Flynn, Wood and Whiten, 2010). Furthermore, in the ghost display condition of Hopper et al.'s (2007) study, no chimpanzees learned how to operate the device in question (the "Pan-pipes" tool-use task, see also Whiten, Horner \& de Waal, 2005). Although Hopper et al. (2007) tested chimpanzees individually in the ghost control condition, which could potentially confound comparisons with live demonstrations, Hopper (2008) reported a replication of this finding with chimpanzees tested in social groups, finding once again that no chimpanzee learned to use the Pan-pipes from a ghost display.

Interestingly, the performance of the chimpanzees with regard to the ghost display contrasts quite strikingly with the performance of young children in an equivalent test using the same task. Hopper et al. (2010) found that up to $34 \%$ of three- to four-year-old children presented with the Pan-pipes apparatus learned how to operate it in a ghost display condition, although success in the live demonstration was considerably higher (77\%).

The apparent absence of emulative learning by chimpanzees in these Pan-pipes studies is difficult to reconcile with the portrayal of chimpanzees as product-oriented copiers. We suspect that the baseline level of task difficulty (see Whiten, McGuigan, Marshall-Pescini \& Hopper, 2009; Hopper et al., 2010), and the transparency of task affordances (see Horner and Whiten, 2005) may well be key factors. Previous studies which have reported emulative learning by chimpanzees (reviewed by Tennie et al., 2009) have employed tasks for which the physical properties and affordances of the task may be more clearly apparent, whereas the mechanism underlying the Pan-pipes task may be more opaque to chimpanzees. Consistent with this, using a simpler task (left/right sliding door), Hopper, Lambeth, Schapiro and Whiten (2008) found evidence for emulative learning by chimpanzees from a ghost display, although it is worth noting that direction copying in the ghost condition was very short-lived 
in contrast with that observed in a corresponding live condition with demonstration provided by a conspecific.

\section{High Fidelity Behavioral Transmission in Chimpanzees}

Although we believe the ghost control studies by Hopper and colleagues indicate that, at least for some tasks, chimpanzees show process-oriented copying, Tennie and colleagues (e.g. Tennie et al., 2009; Tennie, Call \& Tomasello, 2010) have maintained that chimpanzees solve the Pan-pipes task, and show a tendency to match the demonstrator's method, through emulative rather than imitative learning. However, regardless of the mechanisms believed to be responsible, it is undisputed that the behaviors involved can transmit between chimpanzees with remarkably high fidelity (Tennie et al., 2009). Horner et al. (2006), using a lift/slide door apparatus, found that chimpanzees were capable of copying the actions of conspecific models with such fidelity that the two alternative techniques were each faithfully transmitted along chains of task-naive chimpanzees, one of which was four "generations" long, and the other five. The remarkable copying by chimpanzees has also been shown to allow introduced behavioral variants to spread through social groups under more naturalistic conditions, creating captive behavioral traditions (Whiten et al., 2005; Bonnie, Horner, Whiten \& de Waal, 2007; Whiten, Spiteri, Horner, Bonnie, Lambeth, Schapiro, \& de Waal, 2007). Perhaps even more strikingly, it has also been shown that the power of these social influences is such that chimpanzees will continue to use socially-learned behaviors even when they are redundant (Price, Lambeth, Schapiro \& Whiten, 2009), or when the learned behavior causes them to go against personal preferences (Hopper, Schapiro, Lambeth \& Brosnan, 2011). Consequently, it would appear that, whatever the mechanism involved, for these tasks, chimpanzees seem capable of the sort of high fidelity transmission proposed by Tennie et al. (2009) to be vital for cumulative culture.

\section{Human Emulative Learning}

Studies of social learning in humans which incorporate ghost display and/or end state conditions (many of which have been carried out with children) confirm that emulation learning can result in high fidelity transmission. In Hopper et al.'s (2010) study using the Pan-pipes, children showed a marked tendency to use the method they were shown, whether this was by live demonstration or ghost display. Furthermore, in the simpler sliding door task reported in Hopper et al. (2008), children's matching to the ghost displays, in contrast to the chimpanzees', persisted beyond the first trial. Other studies using different tasks reinforce these conclusions. Thomson and Russell (2004), Huang and Charman (2005), and Subiaul, Lurie, Romansky, Klein, Holmes and Terrace (2007) all found evidence of learning from ghost conditions, in children aged between 14 months (Thomson and Russell, 2004) and four years (Subiaul et al., 2007).

With regard to end state displays, Huang, Heyes and Charman (2002) found that 19month old infants who observed the initial state and end state of Meltzoff's (1995) tasks produced as many target actions as those that observed a full demonstration or a failed attempt demonstration. Using a pyramid-building task, Murphy and Wood $(1981 ; 1982)$ found that four- to eight- year old children were able to make use of photographs of the intermediate stages of construction of this object, resulting in considerably higher success in these conditions compared with a control group not shown these materials.

\section{Non-Imitative Cumulative Culture in Humans}

Given that emulation learning can result in high fidelity diffusion, there is no particular reason to believe that humans should be restricted to imitative learning when it comes to cumulative culture. Information about products should in principle be sufficient to allow for 
ratchet-like learning effects. Consistent with this, we (Caldwell \& Millen, 2009) have now shown that this is indeed the case in a study of cumulative culture in adult humans.

Caldwell and Millen's (2009) study represented a direct attempt to test whether cumulative cultural evolution could occur even when adult human participants are deprived of opportunities for learning by imitation and teaching, both suggested to be critical for cumulative culture (Galef, 1992; Tomasello et al, 1993; Tomasello, 1999). In a previous study, Caldwell and Millen (2008) had presented participants with simple tasks, finding that solutions to these tasks improved over laboratory "generations" of participants, with later participants benefiting from opportunities to learn from individuals tested before them. Caldwell and Millen (2009) made use of one of these tasks (paper airplane building) in order to test hypotheses regarding the learning mechanisms necessary for this observed cumulative improvement. Participants were given the goal of building a plane to fly as far as possible. Participants acted as a member of a chain of ten individuals, each of whom built their airplane after gaining information from the prior step in the chain. Caldwell and Millen (2009) manipulated the availability of opportunities for imitation (observation of other participants building), emulation (opportunities to inspect the completed products), and teaching (receiving verbal instruction from other participants), finding that each of these sources of information was independently sufficient for participants to show cumulative culture. It seems therefore that cumulative learning can be supported by purely productoriented copying, although it remains to be seen whether such an effect will extend to other, more complex (or perhaps less familiar), tasks.

\section{End State Copying in Adult Humans: An Experimental Test}

Rationale. The aim of the current experiment was therefore to assess the fidelity of product-oriented copying in a task known to elicit cumulative learning effects in humans. Although it may be clear that high fidelity transmission is possible on the basis of emulation learning, and that cumulative culture can be elicited by emulation learning alone, the more direct relationship between high fidelity transmission and cumulative culture remains unclear. Caldwell and Millen (2009) showed that cumulative culture could arise from emulative learning. However, it could be the case that high fidelity transmission was involved, but that this was possible from end state information. Alternatively it could be the case that high fidelity copying was not involved in transmission, but that cumulative learning effects could nonetheless arise. This latter possibility is plausible since cumulative culture necessarily depends on a balance between social learning and innovation (Tomasello, 1999). Thus the improvements in performance over generations documented by Caldwell and Millen (2008; 2009) might actually have arisen as a consequence of relatively low fidelity transmission.

To address this question, we tested adult humans using a spaghetti tower building task, for which Caldwell and Millen (2008) had identified cumulative improvement in tower height over learner generations (see also Caldwell \& Millen, 2010). Furthermore, it appears that when participants copy in the context of this task it is used in a relatively strategic way, driven by a motivation to perform well in terms of the measured goal. In Caldwell and Millen's (2010) study, there was evidence of greater within-chain similarity in a condition which introduced greater uncertainty about the final height measurement, compared with the (standard) immediate measure. In a follow-up study, Caldwell and Eve (in preparation) deliberately placed heavy emphasis on the goal measure of tower height, making this highly salient (demonstration towers were displayed alongside a tape measure and a sign clearly displaying the tower's exact height), and incorporating a payment structure whereby participant fees were directly and steeply related to the final height. The intention was to determine whether participants' apparent trade-offs between social information and innovation were truly motivated by a desire to perform well on the task. Once again, there 
was evidence of similarity between participants' towers and those they were shown, with greater reliance placed on the social information in a group who were led to believe that innovation might be risky. Consequently, we know that participants employ both social learning and innovation in taking part in this task, and that these are used relatively strategically in an apparent attempt to maximize final scores.

Here, we used this task to conduct a direct test of the strength of copying from end states, extending to a comparison between a condition involving direct observation of finished products, and another which provided only indirect end state information (a photograph of a finished product). Strictly speaking, the studies conducted by Caldwell and Millen $(2008 ; 2009 ; 2010)$ were not direct tests of copying, since there was no controlled manipulation of the task solutions to which participants were exposed (only variation in the spontaneous solutions of other participants). However, Caldwell and Millen (2008; 2010) did find evidence of some copying by analyzing the similarity of solutions by participants from the same chain, compared with those from different chains.

The current experiment therefore represents a more controlled test of copying effects, as we have experimentally manipulated the designs to which participants are exposed, exploiting the logic of 'two-action' experiments (e.g. Whiten et al., 2005). This has permitted a more objective feature-based analysis of participants' designs in order to document the presence or absence of specific elements of the two alternative demonstration towers. Importantly, however, it should be noted that the goal given to participants was identical to the goal given to the participants in the studies of cumulative culture using this task (Caldwell $\&$ Millen, 2008; 2010). Thus, the participants were instructed simply to build their own tower as tall as possible, and any matching to the demonstrated tower design therefore arose as a consequence of participants' own decisions about how best to approach the task.

\section{Method}

Participants. Eighty adult human participants took part in the study, all of whom were recruited on campus at the University of Stirling, United Kingdom. They took part in return for a research participation course credit (for Psychology undergraduates only) or a $£ 3$ participation fee. Fifty-one females and 29 males with a mean age of 22.9 years $(\mathrm{SD}=8.5$, range 18-62 years) took part. Ethical approval for this research was provided by the University of Stirling Psychology Ethics Committee. The procedure was explained to all participants in advance, and each gave written consent to participate.

Apparatus. Each participant was given one $500 \mathrm{~g}$ packet of spaghetti, plus approximately $80 \mathrm{~g}$ of modeling clay (Early Learning Centre "Modelling Material").

Design and procedure. Participants were informed that the goal of the spaghetti tower building task was simply to build a tower as tall as possible using the spaghetti and modeling clay. They were also informed that they would be shown the solutions of two previous participants.

In the 'live' end state condition, the participant arrived to find two spaghetti towers already completed, both of the same design, standing in the testing area. Although they were told that these towers had been built by previous participants, they had in fact been built by the experimenter to a specific design. In the 'photo' end state condition, participants were simply shown two photographs of spaghetti towers, displayed on the screen of a laptop computer, the participants again being led to believe that these were the efforts of the previous participants in the experiment. However, as in the live condition, these towers had also been built to a specific design by the experimenter. Additionally, in the photo end state condition, the towers were shown alongside a familiar object of typical size (a mug), 
providing some information about scale. In both live and photo conditions, the participants were split into two further conditions (with 20 participants per sub-condition), determining which of two alternative tower designs they were shown.

The two alternative tower designs were selected because they represented fairly typical tower types, based on previous experiments using this task, which relied solely on participants' spontaneous design choices, without interference from designs created by an experimenter (Caldwell \& Millen, 2008; 2010). These two designs were also distinctly different, with several features that could be identified to distinguish them. This permitted an objective coding scheme to be developed, based on these contrasting features (Table 1). The tower types were labeled as 'Cubic' and 'Tripod'. Examples of each are shown in Figure 1. Demonstration towers were not built to precise heights, but since they were built to particular designs, the height of demonstration towers was relatively consistent. Cubic-type demonstration towers were approximately $44 \mathrm{~cm}$ tall, and tripod-type demonstration towers were approximately $54 \mathrm{~cm}$ tall. Further information about the differences between their features, and the coding scheme used, is provided below in the Data Coding section. Following five minutes' exposure to the previous designs, participants were informed that they would be given a further five minutes in which to build their own tower. At the end of this period the experimenter took a photograph of the completed tower.

Table 1. The coding scheme used to quantify the similarity of participants' towers to the two demonstration tower types.

\begin{tabular}{|c|c|c|}
\hline Tower Feature & Cubic & Tripod \\
\hline Base contact & $\begin{array}{l}\text { Modeling clay and spaghetti } \\
\text { (1) }\end{array}$ & Modeling clay only (1) \\
\hline Base shape & Square (1) & Triangular (1) \\
\hline Lower level structure & $\begin{array}{l}\text { Vertical from plasticine } \\
\text { contact points, not } \\
\text { converging to single point } \\
\text { (1) }\end{array}$ & $\begin{array}{l}\text { Approximately vertical } \\
\text { converging to single point } \\
\text { (1) }\end{array}$ \\
\hline Upper level structures & $\begin{array}{l}\text { Horizontal joins between } \\
\text { vertical uprights }(0.5) \text {, and } \\
\text { vertical joins into single } \\
\text { point }(0.5)\end{array}$ & $\begin{array}{l}\text { Single vertical element as } \\
\text { highest level (0.5), with } \\
\text { any/all upper levels as } \\
\text { single verticals }(0.5)\end{array}$ \\
\hline
\end{tabular}

Note. Numbers indicate the points attributed to towers displaying those features as their Cubic and Tripod feature scores.

Data coding. Towers built by participants were coded according to the features they had in common with the two alternative demonstration towers. Four aspects were coded as either cubic-like, or tripod-like (Table 1). All photographs were coded according to this scheme by two raters working independently. Their ratings showed high concordance, suggesting that the scores could be assigned with high reliability using this coding scheme (Spearman's Rho for cubic features: $r=.967, N=80, p<.0005$; for tripod features: $r=.973$, $N=80, p<.0005)$. 
Figure 1. The tower types shown to participants. Participants either saw two examples of the Cubic design (a), or two examples of the Tripod design (b).
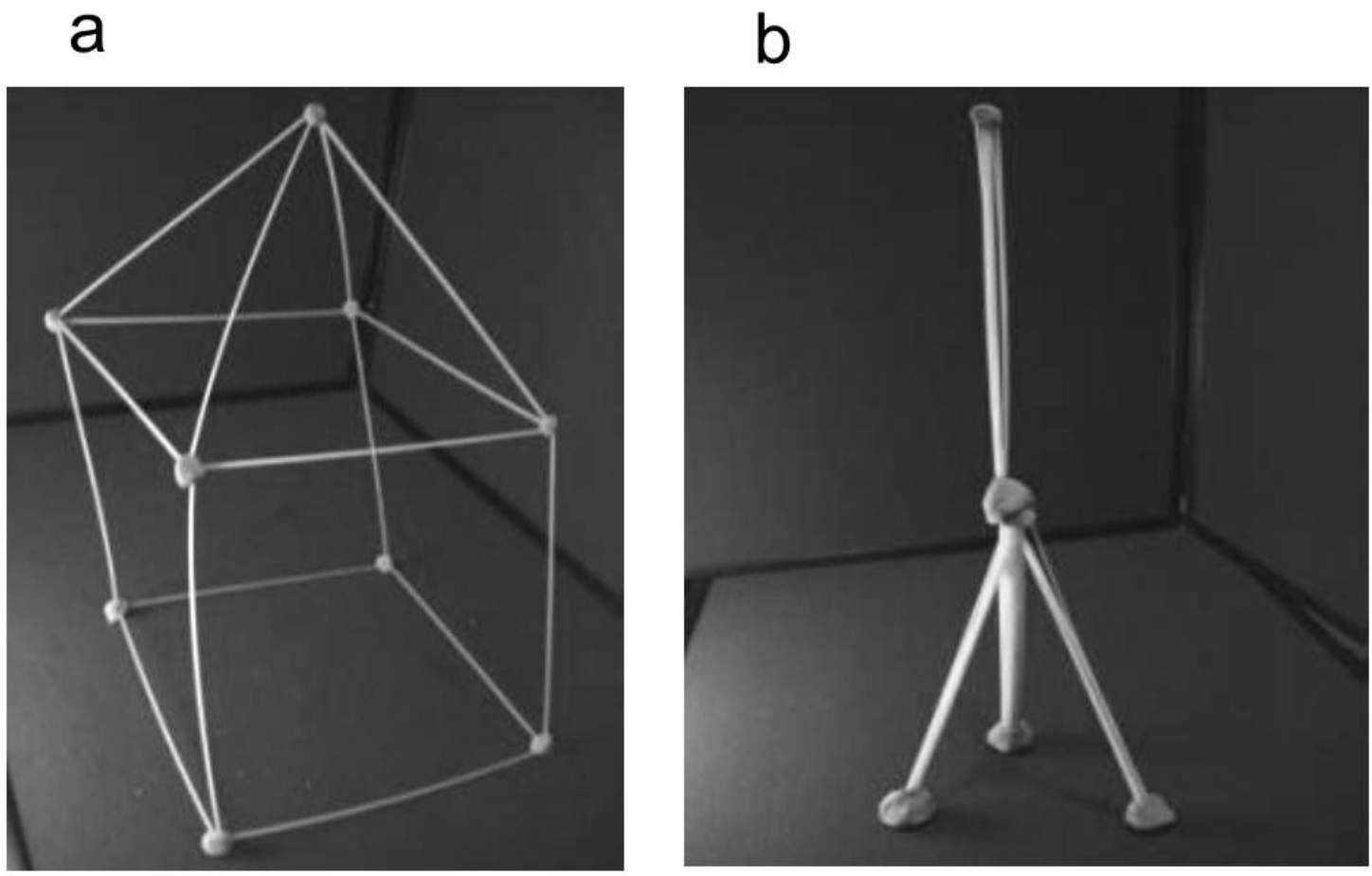

\section{Results}

Matching to Demonstration Towers. Scores using the coding scheme described above were not normally distributed, so non-parametric statistics were applied throughout. In order to test the influence of demonstration tower types, all towers were given a score to indicate the proportion of features they had in common with a given tower type, in relation to the total number of features that they had in common with either tower type. Thus, proportional scores were calculated as: cubic-type features/(cubic-type features + tripod-type features). Scores calculated in this way therefore vary between a minimum of 0 (indicating a tower with only tripod-type features), and a maximum of 1 (indicating a tower with only cubic-type features), with 0.5 representing a tower with an equal number of cubic and tripod-type features. Figure 2 displays the median cubic proportion scores, calculated in this way, for the four different demonstration conditions.

In the cubic tower demonstration conditions, the median cubic proportion of participants' towers was 1.00 for the live condition (first quartile $=0.69$, third quartile $=1$ ), and 1.00 for the photo condition (first quartile $=0.81$, third quartile $=1$ ). In the tripod tower demonstration conditions, the median cubic proportion was 0.08 for the live condition (first quartile $=0$, third quartile $=0.44$ ), and 0.08 for the photo condition (first quartile $=0$, third quartile $=0.60$ ). 
Figure 2. Median proportion of cubic to tripod tower type features in participants' towers in live and photo end-state conditions. Error bars indicate first and third quartiles. $N=20$ in each of the four conditions.

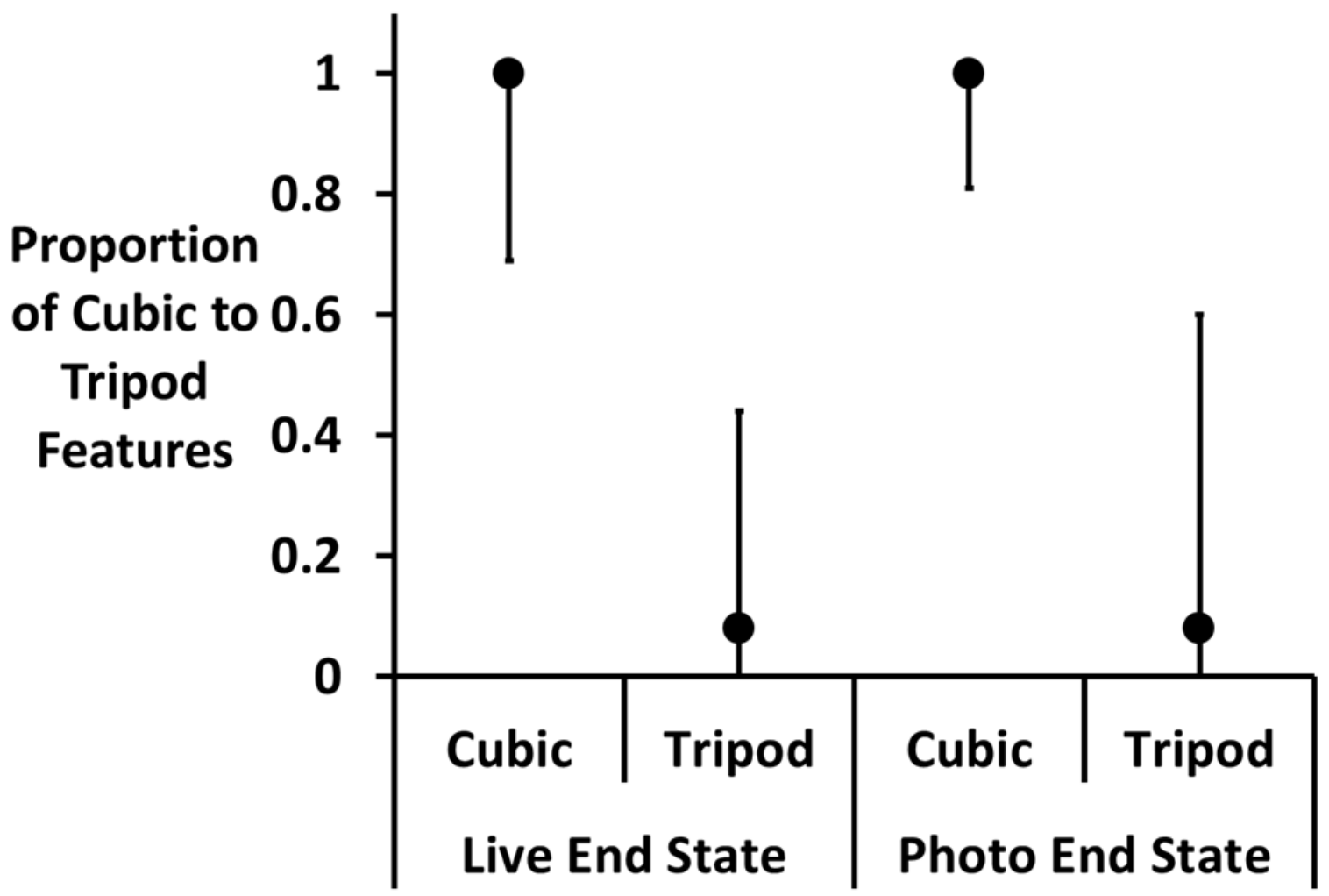

In the cubic tower demonstration conditions, there was no difference in the proportion of cubic to tripod features in towers made by participants in the live cubic and photo cubic conditions (Mann-Whitney $U$ test: $U=196.5, n_{1}=20, n_{2}=20, p=.907$ ). Towers from the tripod tower demonstration conditions also did not differ in the proportion of cubic to tripod features, across the live tripod and photo tripod conditions (Mann-Whitney $U$ test: $U=175$, $\left.n_{1}=20, n_{2}=20, p=.484\right)$. Scores from live and photo conditions were therefore combined when comparing cubic and tripod conditions.

Participants who had seen cubic-type towers produced towers with a significantly higher proportion of cubic to tripod features, compared with those who had seen tripod-type towers (Mann-Whitney $U$ test: $U=145.5, n_{1}=40, n_{2}=40, p<.0005$ ), indicating a high level of matching to the demonstration towers (see Figure 2).

Tower Heights. Tower heights were also non-normally distributed and were analyzed using nonparametric statistics. Towers built in the live cubic demonstration condition had a median height of $51.5 \mathrm{~cm}$ (first quartile $=40.5$, third quartile $=53.3$ ). Towers in the photo cubic demonstration condition had a median height of $50.8 \mathrm{~cm}$ (first quartile $=35.5$, third quartile $=53.0$ ). The median height of towers in the live tripod demonstration condition was $77.8 \mathrm{~cm}$ (first quartile $=69.0$, third quartile $=83.5$ ). For the photo tripod demonstration condition, the median height was $71.5 \mathrm{~cm}$ (first quartile $=52.5$, third quartile $=78.5$ ).

There was no significant difference in height between towers built in the live cubic and photo cubic conditions (Mann-Whitney $U$ test: $U=197.5, n_{1}=20, n_{2}=20, p=.946$ ). There was also no significant difference in height between towers built in the live tripod and photo tripod conditions (Mann-Whitney $U$ test: $U=141, n_{1}=20, n_{2}=20, p=.110$ ). Scores from live and photo demonstration conditions were therefore combined for the purpose of 
comparing towers built in the cubic and tripod conditions. There was a difference in height between towers built in the cubic and tripod conditions (Mann-Whitney $U$ test: $U=291, n_{1}=$ $\left.40, n_{2}=40, p<.0005\right)$, with tripod condition towers being significantly taller. The demonstration towers in the tripod condition were around $10 \mathrm{~cm}$ taller than those in the cubic condition (see Methods). However, participants in the cubic condition built towers that were no different in height from their demonstration towers (One sample Wilcoxon test against 44cm baseline: $Z=1.560, p=.119$ ), whereas those in the tripod condition built towers that were significantly taller than their demonstration towers (One sample Wilcoxon test against 54cm baseline: $Z=3.408, p=.001$ ).

Amongst the towers built in the cubic tower demonstration conditions, there was a significant negative relationship between the number of cubic features (absolute scores from 0 to 4, see Table 1) and height (Spearman's Rho: $r=-0.319, N=40, p=.045$ ). So in the cubic condition, towers that closely matched the demonstration towers were shorter than those that did not. The reverse was the case for the tripod tower demonstration condition, because in the tripod condition there was a significant positive relationship between the number of tripod features and height (Spearman's Rho: $r=0.539, N=40, p<.0005$ ).

Despite this, the absolute number of features matching the demonstration towers did not differ across the tower type conditions. Towers from the cubic demonstration conditions had a median score of 3.5 cubic features (out of a possible 4, see Table 1, first quartile $=2.1$, third quartile $=3.5$ ). Towers from the tripod condition had a median score of 3 tripod features (first quartile $=1.9$, third quartile $=3.9$ ). There was no significant difference between the tower type conditions in terms of the absolute number of features matching the demonstration towers (Mann-Whitney $U$ test: $U=709, n_{1}=40, n_{2}=40, p=.375$ ).

\section{Discussion}

We found a high degree of matching to the tower construction style in both the live and photo conditions. The degree of matching is sufficiently striking that it is important to emphasize once more that the participants in our experiment were never instructed to copy the towers that they were shown; the explicit goal was simply to build a tower as tall as possible. This relatively blinkered copying - only matching the tower form shown and not exploring alternatives - is reminiscent of the phenomenon of "overimitation" (Lyons, Young \& Keil, 2007). Lyons et al. (2007) describe overimitation as, "persistently reproducing ... unnecessary actions" (p19751). Studies of this phenomenon have so far shown that both children (Horner \& Whiten, 2004; Lyons et al., 2007; Lyons, Damrosch, Lin, Macris, \& Keil, 2011; McGuigan, Whiten, Flynn \& Horner, 2007; Nielsen and Tomaselli, 2010) and adults (McGuigan, Makinson \& Whiten, 2011) are susceptible to copying irrelevant aspects of an experimenter's demonstration in social learning experiments. In the current study we did not intentionally incorporate irrelevant elements as was done in these previous studies, however, since our study concerned learning from end states, it would not have been possible to incorporate irrelevant actions as the previous studies have done. All the same, although we did not set out to introduce superfluous structural elements to either design, it is clear that the cubic design was relatively inefficient with regard to the goal of maximizing height. The results concerning the heights of the towers built across conditions show quite clearly that the tripod design was a more effective approach, and yet participants exposed to the cubic design copied with an equivalent degree of fidelity.

How can such apparently counterproductive copying be consistent with cumulative culture? As mentioned earlier, cumulative culture is dependent on both social learning and innovation, and as a consequence we might expect to see relatively low fidelity copying in a 
task such as this, where it is theoretically possible to perform considerably better than the previous solutions shown. The high levels of matching observed in the current experiment, especially with regard to the less effective of the two designs, might be expected to inhibit ratcheting. However, it is worth pointing out that although our cubic design was less effective than the tripod design, it was nonetheless a perfectly viable solution to the task. From the participants' point of view this would have been self-evident from the examples shown. In the absence of any information about the relative effectiveness of alternative designs, copying may have been regarded simply as less risky than innovation, the outcome of which would have been less predictable. The apparent strategic shifts between social learning and innovation observed in other studies involving this task, mentioned in the introduction (Caldwell \& Millen, 2010; Caldwell \& Eve, in preparation), provide some support for this interpretation. Indeed, it is possible that participants in our experiment interpreted the absence of any examples of a contrasting type as an indication that alternative approaches were likely to be less effective, since the previous participants had apparently decided to avoid them. Such inferences about the knowledge and rationality of the demonstrator may go some way towards explaining other results in the literature on "overimitation", particularly given the otherwise rather counterintuitive finding that such tendencies appear to increase with age (McGuigan et al., 2007; McGuigan \& Whiten, 2009; McGuigan et al., 2011).

Interpreting high fidelity copying as cautious, rather than simply imperceptive, may be key to understanding its relationship to cumulative culture. As Tomasello (1999) has noted, it is faithful transmission which prevents "slippage backwards", providing the stabilizing component of the ratchet. The cautious approach taken by our participants therefore seems entirely consistent with this view. All the same, this remains a relatively speculative interpretation of the current data, and it is possible that participants' behavior in the current study was driven by lower-level mechanisms, such as priming of particular shapes and/or structures.

At any rate, it seems clear that participants were not simply copying as a consequence of normative pressure. Deutsch and Gerard (1955) distinguished "normative" from "informational" effects, defining normative social influence as "an influence to conform with the positive expectations of another" (p. 629), and informational social influence as "an influence to accept information obtained from another as evidence about reality" (p. 629). Deutsch and Gerard (1995) suspected that both effects were at work in typical studies of conformity involving erroneous responses given by confederates. Described in terms of such studies, if affected purely by normative influence a participant would shift their response in line with those of the confederates in spite of a private belief that the confederates were mistaken. In contrast, if purely informational influence was involved, a participant would alter their responses because they were concerned only about making the correct response, and had concluded that the confederates must be right. Relating this to our experiment, our participants never met the designers of the towers that they were shown, and would have been aware that their own design, if shown to others, would be equally anonymous. There was therefore little or no motivation to override one's own intuition in preference for adhering to what was believed to be an inferior demonstration design.

To return to the theoretical points raised in the introduction, we can now further evaluate the proposal of Tennie et al (2009). As already noted, we do not agree that imitation (copying of processes of behavior) is key to cumulative culture, since chimpanzees appear capable of copying processes, and humans appear capable of cumulative culture in the absence of information about actions. On the basis of our results in the current experiment, we would suggest that the reason that humans do not appear to require imitation in order to engage in cumulative culture is not because high fidelity transmission is unimportant to 
cumulative culture, but rather because imitative learning is not always necessary for high fidelity transmission.

An important caveat must be added to this point however. Clearly, under many circumstances, imitative learning is absolutely crucial. Many behaviors (gestural communicative conventions, for example) leave no physical trace, and involve no movement other than the bodily action itself. The notion of an end state, or ghost display, is therefore meaningless in such contexts, and it is not possible to learn such behaviors through emulation.

Also, as noted in the introduction, the transparency of the task is also very likely to increase reliance on imitation. Our task was highly transparent, in the sense it was possible to infer the intermediate steps needed to achieve the goal without necessarily observing them. But many other tasks are far more opaque in this respect. Hopper et al. (2008, 2010) have argued that imitation and emulation tend to be applied strategically, where tasks are opaque and transparent respectively (see also Acerbi, Tennie \& Nunn, 2010). Hopper et al. (2010) found that children who saw a human model demonstrate the Pan-pipes task were more successful than those who saw a ghost display, suggesting that imitative learning was very helpful for this cognitively opaque task. It appears therefore that human children are capable of using different social learning mechanisms (imitation or emulation) depending on the task complexity and information available to them (Nielsen, 2006). But since chimpanzees also appear to be flexible in their use of social learning mechanisms (e.g. Hopper et al., 2007; 2008; Horner \& Whiten, 2005) this still fails to fully explain the apparent absence of cumulative culture in chimpanzees.

The cause of the obvious differences in the richness of culture between humans and chimpanzees therefore remains an open debate, and we believe that alternative explanations should be considered. The apparent conservatism exhibited by chimpanzees within studies of social learning may provide an intriguing insight. Chimpanzees appear to show a striking tendency to perseverate with learned responses, often in the face of exposure to, or even experience of, more effective alternatives (Marshall-Pescini \& Whiten, 2008; Hrubesch, Preuschoft \& van Schaik, 2009; Price et al., 2009; Hanus, Mendes, Tennie \& Call, 2011; Hopper et al., 2011).

It is important to note that the conservatism reported in chimpanzees appears very different from the "overimitation" observed in studies of social learning in humans, even though it may sometimes entail persistence with a socially learned solution in the face of individual experience of a more effective alternative (e.g. Hopper et al., 2011), which could be regarded as reminiscent of overimitation. As noted above, the high fidelity copying documented in the current experiment (even with regard to a relatively inefficient demonstration design) probably arises as a result of a genuine motivation to achieve an adequate, if not outstanding, score on the goal measure. Although it was not within the scope of the current study, it would be possible in future studies to show participants a relatively poor demonstration tower (e.g. cubic design) prior to their first attempt, and then show them a more effective solution (e.g. tall tripod, similar to those built by many of the participants in the tripod condition) to determine whether they would switch strategies. We would predict that human participants would readily switch to the more effective alternative. And yet, it is exactly this kind of judicious behavioral flexibility that chimpanzees appear to struggle with.

However, it remains to be seen whether such effects apply across other primates, and indeed other nonhuman animals. It is possible that the conservatism of chimpanzees may not provide an explanation in and of itself, but understanding the reasons why it occurs could perhaps facilitate our understanding of the uniqueness of human cumulative culture. 
To conclude, we believe that human cumulative culture, and high fidelity copying, can be supported by mechanisms like emulation, as well as imitation and teaching. Further explicit testing of such effects is warranted.

\section{Acknowledgements}

C.A.C., K.S. \& C.L.E. were funded by a research grant from the Economic and Social Research Council (RES-062-23-1634). L.M.H. was supported by an NSF CAREER grant (SES 0847351, awarded to Dr. Sarah Brosnan), during the writing of the paper.

\section{References}

Acerbi, A., Tennie, C. \& Nunn, C. L. (2010). Modelling imitation and emulation in constrained search spaces. Learning and Behavior, 39, 104-114.

Bonnie, K. E., Horner, V., Whiten, A., \& de Waal, F. B. M. (2007). Spread of arbitrary conventions among chimpanzees: a controlled experiment. Proceedings of the Royal Society B: Biological Sciences, 274, 367-372.

Boyd, R. \& Richerson, P. J. (1996). Why culture is common but cultural evolution is rare. Proceedings of the British Academy, 88, 77-93.

Byrne, R. W. (1998). Comment on 'Chimpanzee and human cultures' by Boesch \& Tomasello. Current Anthropology, 39, 604-605.

Caldwell, C. A. \& Millen, A. E. (2008). Experimental models for testing hypotheses about cumulative cultural evolution. Evolution and Human Behavior, 29, 165-171.

Caldwell, C. A. \& Millen, A. E. (2009). Social learning mechanisms and cumulative cultural evolution: is imitation necessary? Psychological Science, 20, 1478-1483.

Caldwell, C. A. \& Millen, A. E. (2010). Conservatism in laboratory microsocieties: unpredictable payoffs accentuate group-specific traditions. Evolution and Human Behavior, 31, 123-130.

Call, J., Carpenter, M. \& Tomasello, M. (2005). Copying results and copying actions in the process of social learning: chimpanzees (Pan troglodytes) and human children (Homo sapiens). Animal Cognition, 8, 151-163.

Deutsch, M. \& Gerard, H. B. (1955). A study of normative and informational social influences upon individual judgment. Journal of Abnormal and Social Psychology, 51, 629-36.

Galef, B. G. (1992). The question of animal culture. Human Nature, 3, 157-178.

Hanus, D., Mendes, N., Tennie, C., \& Call, J. (2011). Comparing the performance of apes (Gorilla gorilla, Pan troglodytes, Pongo pygmeaus) and human children (Homo sapiens) in the floating peanut task. PloS ONE, 6, e19555.

Hill, K., Barton, M. \& Hurtado, A. M. (2009). The emergence of human uniqueness: characters underlying behavioral modernity. Evolutionary Anthropology, 18, 187-200.

Hopper, L. M. (2010). 'Ghost' experiments and the dissection of social learning in humans and animals. Biological Reviews, 85, 685-701.

Hopper, L. M. (2008) Dissection of observational learning among chimpanzees (Pan troglodytes) and children (Homo sapiens). PhD Thesis, University of St Andrews, UK [http://hdl.handle.net/10023/436].

Hopper, L. M., Flynn, E. G., Wood, L. A. N. \& Whiten, A. (2010). Observational learning of tool use in children: investigating cultural spread through diffusion chains and learning 
mechanisms through ghost displays. Journal of Experimental Child Psychology, 106, 82-97.

Hopper, L. M., Lambeth, S. P., Schapiro, S. J. \& Whiten, A. (2008). Observational learning in chimpanzees and children studied through 'ghost' conditions. Proceedings of the Royal Society B, 275, 835-840

Hopper, L. M., Schapiro, S. J., Lambeth, S. P., and Brosnan, S. F. (2011). Chimpanzees' socially maintained food preferences indicate both conservatism and conformity. Animal Behaviour, 81, 1195-1202.

Hopper, L. M., Spiteri, A., Lambeth, S. P., Schapiro, S. J., Horner, V. \& Whiten, A. (2007). Experimental studies of traditions and underlying transmission processes in chimpanzees. Animal Behaviour, 73, 1021-1032.

Horner, V. \& Whiten. A. (2005). Causal knowledge and imitation/emulation switching in chimpanzees (Pan troglodytes) and children (Homo sapiens). Animal Cognition, 8, 164181.

Horner, V., Whiten, A., Flynn, E. \& de Waal, F. B. M. (2006). Faithful replication of foraging techniques along cultural transmission chains by chimpanzees and children. Proceedings of the National Academy of Science, 103, 13878-13883.

Hrubesch, C., Preuschoft, S. \& van Schaik, C. P. (2009). Skill mastery inhibits adoption of observed alternative solutions among chimpanzees (Pan troglodytes). Animal Cognition, 12, 209-216.

Huang, C-T. \& Charman, T. (2005). Gradations of emulation learning in infants' imitation of actions on objects. Journal of Experimental Child Psychology, 92, 276-302.

Huang, C-T., Heyes, C. \& Charman, T. (2002). Infants' behavioral reenactment of "failed attempts": exploring the roles of emulation learning, stimulus enhancement, and understanding of intentions. Developmental Science, 38, 840-855.

Laland, K. N. \& Galef, B. G. (2009). The Question of Animal Culture. Harvard University Press: Cambridge, MA.

Lyons, D. E., Damrosch, D. H., Lin, J. K., Macris, D. M. \& Keil, F. C. (2011). The scope and limits of overimitation in the transmission of artefact culture. Philosophical Transactions of the Royal Society B, 366, 1158-1167.

Lyons, D. E., Young, A. G. \& Keil, F. C. (2007). The hidden structure of overimitation. Proceedings of the National Academy of Sciences of the USA, 104, 19751-19756.

Marshall-Pescini, S. \& Whiten, A. (2008). Chimpanzees (Pan troglodytes) and the question of cumulative culture: an experimental approach. Animal Cognition, 11, 449-456.

McGuigan, N. \& Whiten, A. (2009). Emulation and "overemulation" in the social learning of causally opaque versus causally transparent tool use by 23 - and 30-month-olds. Journal of Experimental Child Psychology, 104, 367-381.

McGuigan, N., Whiten, A., Flynn, E., \& Horner, V. (2007). Imitation of causally opaque versus causally transparent tool use by 3- and 5-year-old children. Cognitive Development, 22, 353-364.

McGuigan, N., Makinson, J. \& Whiten, A. (2011). From over-imitation to super-copying: Adults imitate causally irrelevant aspects of tool use with higher fidelity than young children. British Journal of Psychology, 102, 1-18.

Meltzoff, A. N. (1985). Immediate and deferred imitation in fourteen- and twenty-fourmonth-old infants. Child Development, 56, 62-72.

Meltzoff, A. N. (1995). Understanding the intentions of others: reenactment of intended acts by 18-month-old children. Developmental Psychology, 31, 838-850.

Murphy, C. M. \& Wood, D. J. (1981). Learning from pictures: the use of pictorial information by young children. Journal of Experimental Child Psychology, 32, 279297. 
Murphy, C. M. \& Wood, D. J. (1982). Learning through media: a comparison of 4-8 year old children's responses to filmed and pictorial instruction. International Journal of Behavioral Development, 5, 195-216.

Nielsen, M. (2006). Copying actions and copying outcomes: Social learning through the second year. Developmental Psychology, 42, 555-565.

Nielsen, M. \& Tomaselli, K. (2010). Overimitation in Kalahari Bushman children and the origins of human cultural cognition. Psychological Science, 21, 729-736.

Price, E. E., Lambeth, S. P., Schapiro, S. J. \& Whiten, A. (2009). A potent effect of observational learning on chimpanzee tool construction. Proceedings of the Royal Society B, 276, 3377-3383.

Sanz, C., Call, J. \& Morgan, D. (2009). Design complexity in termite-fishing tools of chimpanzees (Pan troglodytes). Biology Letters, 5, 293-296.

Subiaul, F., Lurie, H., Romansky, K., Klein, T., Holmes, D. \& Terrace, H. (2007). Cognitive imitation in typically-developing 3- and 4-year-olds and individuals with autism. Cognitive Development, 22, 230-243.

Tennie, C., Call, J. \& Tomasello, M. (2009). Ratcheting up on the ratchet: on the evolution of cumulative culture. Philosophical Transactions of the Royal Society B, 364, 2405-2415.

Tennie, C., Call, J. \& Tomasello, M. (2010). Evidence for emulation in chimpanzees in social settings using the floating peanut task. PLoS ONE, 5, e10544.

Tennie, C., Greve, K., Gretscher, H. \& Call, J. (2010). Two-year-old children copy more reliably and more often than nonhuman great apes in multiple observational learning tasks. Primates, 51, 337-351.

Thomson, D. E. \& Russell, J. (2004). The ghost condition: Imitation versus emulation in young children's observational learning. Developmental Psychology, 40, 882-889.

Tomasello, M. (1998). Emulation learning and cultural learning. Behavioral and Brain Sciences, 21, 703-704.

Tomasello, M. (1999). The cultural origins of human cognition. Cambridge, MA: Harvard University Press.

Tomasello, M., Kruger, A. C. \& Ratner, H. H. (1993). Cultural learning. Behavioral and Brain Sciences, 16, 495-552.

Whiten, A. (2011). The scope of culture in chimpanzees, humans and ancestral apes. Philosophical Transactions of the Royal Society, 366, 935-1187.

Whiten, A., Horner, V. \& de Waal, F. B. M. (2005). Conformity to cultural norms of tool use in chimpanzees. Nature, 437, 737-740.

Whiten, A., McGuigan, N., Marshall-Pescini, S. \& Hopper, L. M. (2009). Emulation, imitation, over-imitation and the scope of culture for child and chimpanzee. Philosophical Transactions of the Royal Society of London B, 364, 2417-2428.

Whiten, A., Spiteri, A., Horner, V., Bonnie, K. E., Lambeth, S. P., Schapiro, S. J. \& de Waal, F. B. M. (2007) Transmission of multiple traditions within and between chimpanzee groups. Current Biology, 17, 1038-1043.

Wood, D. (1989). Social interaction as tutoring. In M. H. Bornstein \& J. S. Bruner (Eds.), Interaction in Human Development (pp. 59-80). Hillsdale, New Jersey: Lawrence Erlbaum Associates, Publishers.

Zohar, O. \& Terkel, J. (1991). Acquisition of pine cone stripping behavior in black rats (Rattus rattus). International Journal of Comparative Psychology, 5, 1-6. 